\title{
Critical Resistivity along the Quantum Hall Liquid-Insulator Transition Line
}

\author{
Efrat Shimshoni \\ Department of Mathematics-Physics, Oranim-Haifa University, Tivon 36006, Israel.
}

(October 30, 2018)

\begin{abstract}
The critical resistivity $\rho_{x x}^{c}$ measured along the quantum Hall liquid-insulator transition line indicates a pronounced peak for a critical filling factor $\nu_{c} \sim 1$. The origin of this behavior, which marks the crossover from the high to low magnetic field regime in the phase diagram, is explained in the framework of classical transport in a puddle network model. The proposed scenario is also consistent with the behavior of the critical Hall resistance along the transition line. In addition, a functional form is suggested as a fit for isotherms $\rho_{x x}(\nu)$, to be compared with experimental data in the moderately high field regime.
\end{abstract}

The rich phase diagram of a disordered twodimensional electron system in a perpendicular magnetic field consists of two prominent types of phases, distinguishable by their transport properties at low temperatures $(T)$. These include the quantum Hall (QH) liquid states, characterized by a quantized Hall resistivity $\rho_{x y}$ accompanied by $\rho_{x x} \rightarrow 0$, and insulating states in which $\rho_{x x} \rightarrow \infty$. The transitions between adjacent phases have been extensively studied [1 5]. More recently, there has been much progress in the experimental effort to map the phase diagram in the integer $\mathrm{QH}$ regime [6,7], and thus test the theoretically predicted structure [8]. In particular, a transition line separating the conducting phases from the insulator was identified in the $n-B$ plane (where $n$, the carrier density and $B$, the magnetic field, are the two independent control parameters). Along this line, in the moderately low $B$ regime, direct transitions are observed from $N>1 \mathrm{QH}$ states ( $N$ integer) to the insulator [7]. This behavior, and the overall structure of the diagram, are consistent with a numerical result based on the tight binding model for non-interacting electrons [9].

Comparison of the transport data along the conductorinsulator transition line $\left(n_{c}\left(B_{c}\right)\right)$ in Refs. [6] and [7] indicates significant differences, particularly at low $B$ (where the GaAs hole system studied in Ref. [6] exhibits a $B=0$ metal-insulator transition at a finite $n_{c}$ ). These can be attributed to sample dependent properties. However, the critical resistivity $\rho_{x x}^{c}$ measured along this transition line exhibits a striking common feature (observed in other samples as well 10]): it has a pronounced peak at $\nu_{c} \sim 1$. Here $\nu_{c}$ is the critical value of the Landau level (LL) filling factor $\left(\nu \equiv n \phi_{0} / B\right.$, with $\phi_{0}$ the flux quantum), so that $\nu_{c} \sim 1$ marks the crossover from high to low $B$, where in the latter, more than one LL is significantly occupied. This crossover is also signified by the critical Hall resistivity $\rho_{x y}^{c}$ [7]: for $\nu_{c} \leq 1$ it is quantized at $\rho_{x y}^{c}=h / e^{2}$, while above $\nu_{c} \sim 1$ it exhibits a classical Hall effect $\rho_{x y}^{c} \approx h / \nu_{c} e^{2} \cdot \rho_{x x}^{c}$ saturates to the universal value $\sim h / e^{2}$ in the high $B$ limit where $\nu_{c} \rightarrow 1 / 2$. However, the peak value of $\rho_{x x}^{c}$ is significantly elevated from $h / e^{2}$ and sample dependent $\left(\sim 4 h / e^{2}\right.$ in Ref. [6], $\sim 2 h / e^{2}$ in Ref. [7]).

Since this puzzling behavior of $\rho_{x x}^{c}$ appears to be generic, it calls for a simple explanation of its physical origin. In this paper I show that the observed dependence of $\rho_{x x}$ on $\nu$, and in particular the peak of $\rho_{x x}^{c}$ at $\nu_{c} \sim 1$, follow from a phenomenological model for the transport, formerly introduced to interpret data in the high $B$ limit [11,12. The essential assumption underlying this model is that near the transition from a QH liquid to the insulator, the transport is dominated by narrow junctions connecting QH puddles; the puddles are defined by regions encircled by current carrying channels (edge states), and their typical size is assumed to be larger than a characteristic dephasing length. As a result, the longitudinal resistance of the sample is that of a classical random resistor network, and the Hall resistance is quantized provided all the $\mathrm{QH}$ puddles are at the same filling factor. This scenario successfully explains the experimentally observed quantized Hall insulator phenomenon [4], as well as the $\nu$-dependence of $\rho_{x x}$ [3]. More recently, a similar model for the transport was shown to be consistent with the transport properties at low $B$, in samples indicating a $B=0$ metal-insulator transition 13 .

It is useful to start by showing that in the high $B$ limit, this model implies $\rho_{x x}^{c} \approx h / e^{2}$, regardless of the details of the disorder potential. In this limit only the lowest LL is occupied, hence all the $\mathrm{QH}$ puddles contain a single conducting channel. The longitudinal resistance of the junction $j$ between adjacent puddles in the network is therefore given by the Landauer-Büttiker formula [14]

$$
R_{x x}^{j}=\frac{h}{e^{2}} \frac{\mathcal{R}_{j}}{\mathcal{T}_{j}},
$$

where $\mathcal{T}_{j}=1-\mathcal{R}_{j}$ is the transmission probability across the junction; the Hall resistance $R_{x y}^{j}=h / e^{2}$ for any $j$. As proven in Ref. [11], the global resistivity tensor of the sample conveniently separates into Hall and longitudinal components: $\rho_{x y}=h / e^{2}$, and $\rho_{x x}$ is set by the random network of the resistors $\left\{R_{x x}^{j}\right\}$. Namely, $\rho_{x x} \approx R_{x x}^{p}$, $p$ being the resistor at the percolation threshold of the distribution [15]. The dependence of $\rho_{x x}$ on $T$ and $\nu$ is therefore determined by $\mathcal{T}_{p}(T, \nu)$. For sufficiently high $T$ such that quantum tunneling is neglected [13,

$$
\mathcal{T}_{p}(T, \nu)=f\left(\epsilon_{p}-\epsilon_{F}(\nu)\right), \quad f(\epsilon) \equiv \frac{1}{1+e^{\epsilon / k_{B} T}} ;
$$


here $\epsilon_{F}(\nu)$ is the Fermi level, and $\epsilon_{p}$ is the energy of the saddle point at which the junction $p$ is located. Employing Eq. (11), one obtains

$$
\rho_{x x} \approx \frac{h}{e^{2}} \frac{1-\mathcal{T}_{p}}{\mathcal{T}_{p}}=\frac{h}{e^{2}} \exp \left[\frac{\epsilon_{p}-\epsilon_{F}(\nu)}{k_{B} T}\right] .
$$

It follows immediately that the critical filling factor $\nu_{c}$, defined such that $\rho_{x x}\left(\nu_{c}\right)$ is independent of $T$, obeys $\epsilon_{F}\left(\nu_{c}\right)=\epsilon_{p}$ so that the exponent in Eq. (3) vanishes. Consequently,

$$
\rho_{x x}^{c}=\rho_{x x}\left(\nu_{c}\right) \approx \frac{h}{e^{2}} .
$$

Note that this argument can be extended to account for quantum tunneling across the junctions. Eq. (2) is then generalized to a Sommerfeld expansion of the form

$$
\mathcal{T}_{p}(T, \nu)=f\left(\epsilon_{p}-\epsilon_{F}(\nu)\right)+\sum_{n=1}^{\infty} \alpha_{n}(T) f^{(2 n)}\left(\epsilon_{p}-\epsilon_{F}(\nu)\right),
$$

where $f^{(2 n)}(x)$ is the $2 n$-th derivative of the Fermi function. This yield a $T$-independent expression for $R_{x x}^{p}$ provided all terms in the sum vanish. Since $f^{(2 n)}(x)=$ $-f^{(2 n)}(-x)$, this occurs for $\epsilon_{F}(\nu)=\epsilon_{p}$, and once again $\rho_{x x}^{c} \approx h / e^{2}$. It is also important to notice that the above argument for the universality of $\rho_{x x}^{c}$ is not violated in the case where $\nu_{c}$ is different from $1 / 2$ due to lack of particlehole symmetry in the disorder potential. The only essential assumption is the neglect of mixing with higher LL. An interesting example for a remarkable universality of $\rho_{x x}^{c}$ at arbitrary $\nu_{c}$ is provided by the transitions from a fractional QH liquid to the insulator [2]; this observation will be discussed towards the end of the paper.

The above scenario changes once the magnetic field $B$ (at a given $n$ ) is reduced to a point where states in the second LL are occupied. In terms of the configuration of conducting channels in real space, this corresponds to the formation of closed loops of a second channel at the Fermi level inside the QH puddles. The overall filling factor of the system is given by

$$
\nu=p\left[\left(1-p_{h}\right)+\nu_{h} p_{h}\right]
$$

where $p \equiv A_{l} / A$ is the total area fraction of the conducting liquid, $p_{h} \equiv A_{h} / A_{l}$ is the relative area fraction occupies by higher LL states and $\nu_{h} \geq 2$ their local filling factor. For a smoothly varrying disorder potential, $p_{h}$ can be estimated noting that $p_{h}=r_{2}^{2} / r_{1}^{2}$, where $r_{1(2)}$ is the average radius of the region surrounded by the first (second) LL channel in a typical QH puddle, and

$$
\frac{1}{2} K r_{1}^{2} \approx \frac{1}{2} K r_{2}^{2}+\Delta(B)
$$

$\Delta(B)$ is either the cyclotron gap $\hbar \omega_{c}$ or the Zeeman energy gap for spin-resolved Landau bands, and $K$ is the average curvature of the potential local minima. The critical filling factor $\nu_{c}$ for a transition to the insulator is then expressed as

$$
\nu_{c}=p_{c}\left[1+\frac{\left(\nu_{h}-1\right) r_{2}^{2}}{r_{1}^{2}}\right] \approx p_{c}\left[\nu_{h}-\frac{B_{c}}{B_{0}}\right],
$$

where $p_{c}$ is the percolation threshold of the liquid, $B_{c}$ the critical field, and $B_{0}$ is a sample dependent parameter: $B_{0}=m^{*} c K A_{p} / h e\left(\nu_{h}-1\right), B_{0}=K A_{p} / 2 \pi g \mu_{B}\left(\nu_{h}-1\right)$ for the spin-unresolved and spin-resolved cases, respectively ( $m^{*}$ is the effective mass of the carriers, $A_{p}$ is the typical puddle area at percolation). Note that Eq. (8) is valid for $0 \leq r_{2} \leq r_{1}$, i.e. for $p_{c} \leq \nu_{c} \leq \nu_{h} p_{c}$; for a particlehole symmetric potential $p_{c}=1 / 2$, hence the expression describes an interpolation between $\nu_{c} \sim 1 / 2($ at high $B$ ) and $\nu_{c} \sim \nu_{h} / 2$. Neglecting the occupation of LL with $N>2, \nu_{h} \sim 2$ and the upper limit of this regime is $\nu_{c} \sim 1$. The critical density is then given by

$$
n_{c} \approx \frac{1}{\phi_{0}}\left[B_{c}-\frac{B_{c}^{2}}{2 B_{0}}\right],
$$

which is indeed a reasonable fit to the shape of the critical line $n_{c}\left(B_{c}\right)$ in Ref. [6], for $\nu_{c}<1$.

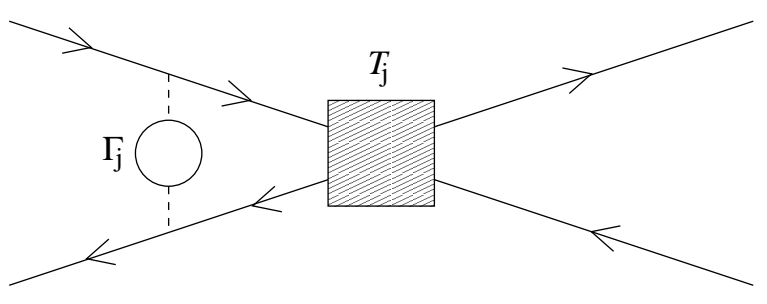

FIG. 1. The configuration of conducting channels near a typical junction connecting adjacent QH puddles.

More interesting is the effect of occupied higher LL states on $\rho_{x x}$ as $\nu_{c} \rightarrow 1$. In the regime $\nu_{c}<1$, corresponding to $p_{h}<1$, these states are localized, forming closed loops inside the QH puddles. A typical junction in the network of conducting channels is schematically depicted in Fig. 1. As $p_{h} \rightarrow 1$, the localized high LL states are coupled to the primary conducting channels via tunneling through occasional weak links (dashed lines in the figure), and thus play the role of resonant scatterers which induce backscattering across the conducting regions. Denoting this backscattering rate close to the $j$ th junction by $\Gamma_{j}$, and neglecting quantum interference between the two reflection paths [16], the effective transmission through the junction is reduced by a factor of $\left(1-\Gamma_{j}\right)$. The junction resistance becomes

$$
R_{x x}^{j}=\frac{h}{e^{2}} \frac{1-\left(1-\Gamma_{j}\right) \mathcal{T}_{j}}{\left(1-\Gamma_{j}\right) \mathcal{T}_{j}}
$$


The entire distribution of resistors in the random network is thus shifted up, and consequently the network resistance (determined as usual by the percolative resistor $\left.R_{x x}^{p}\right)$ is

$$
\rho_{x x} \approx \frac{h}{e^{2}} \frac{1-\left(1-\Gamma_{p}\right) f\left(\epsilon_{p}-\epsilon_{F}(\nu)\right)}{\left(1-\Gamma_{p}\right) f\left(\epsilon_{p}-\epsilon_{F}(\nu)\right)} .
$$

Here I assumed that the dependence of $\Gamma_{p}$ on $T$ is negligible compared to the transmission across the junction, which involves an energy barrier. Eq. (11) clearly implies that the $T$ dependence disappears at a single value of the filling factor $\nu_{c}$ where $\epsilon_{F}\left(\nu_{c}\right)=\epsilon_{p}$, as in the high $B$ limit. However, the value of the critical resistivity thus defined is modified:

$$
\rho_{x x}^{c} \approx \frac{h}{e^{2}} \frac{1+\Gamma_{p}}{1-\Gamma_{p}}>\frac{h}{e^{2}}
$$

For $\nu_{c} \leq 1$, the area fraction $p_{h}$ increases, implying that $\Gamma_{p}$ is obviously an increasing function of $\nu_{c}$ (the explicite behavior of $\Gamma_{p}\left(\nu_{c}\right)$ depends on details of the disorder potential). As a result, $\rho_{x x}^{c}$ grows with $\nu_{c}$ towards the limit case $\nu_{c} \sim 1$.

Another characteristic of the $\nu_{c}<1$ (high $B$ ) regime is that in the entire range of parameters where the classical transport scenario is valid, the Hall resistance maintains the quantized value $\rho_{x y}=h / e^{2}$. This follows from the fact that the Hall resistance of each junction as depicted in Fig. 1 is determined by the chemical potential difference between parallel right and left moving conducting channels, which is not affected by the modified transmission probability and yields $R_{x y}^{j}=h / e^{2}$ for any $j$.

The case where $\nu_{c} \sim 1$ marks a crossover to a low $B$ regime. From Eq. (8) (assuming $p_{c} \approx 1 / 2, \nu_{h} \approx 2$ ), $\nu_{c}=1$ corresponds to $r_{2} \approx r_{1}$, which means that the average size of regions with a local filling fraction $\nu \geq 2$ coincides with the average size of the conducting regions [17]. Beyond this threshold value of $\nu_{c}$, higher LL states are delocalized, adding conducting channels to the percolating (or nearly percolating) network. As a consequence, the resistance of a typical junction $R_{x x}^{j}$ acquires a form similar to Eq. (10), reduced by an overall factor of $1 / N$, where $N$ is the number of conducting channels that are clamped together. Note that $\Gamma_{j}$ then describing scattering to partially occupied LL higher then $N$. The resulting critical value of the global longitudinal resistivity is then reduced compared to its value at $\nu_{c} \sim 1$, and is given by an expression of the form

$$
\rho_{x x}^{c} \approx \frac{h}{N e^{2}} \frac{1+\Gamma_{p}}{1-\Gamma_{p}} .
$$

Here both $N$ and $\Gamma_{p}$ depend on $\nu_{c}$ in a complicated manner, hence the actual behavior of $\rho_{x x}^{c}$ at $\nu_{c} \gg 1(B \rightarrow 0)$ is expected to be strongly sample dependent. Indeed, in Ref. [7] $\rho_{x x}^{c} \rightarrow 0$ in this limit, while in Ref. [6] it saturates back to $\rho_{x x}^{c} \sim h / e^{2}$. However, in proximity to the threshold $\nu_{c} \sim 1$ the generic trend is a reduction of $\rho_{x x}^{c}$ when $\nu_{c}$ is increased beyond unity. It therefore follows that this crossover between the high and low $B$ regimes is indicated by a peak in the critical resistance.

The increasing number of conducting channels 'clamped' together in the low $B$ regime also modifies the Hall resistance, since $\left\{R_{x y}^{j}\right\}$ are no longer identical. As argued in Ref. [11], the pure transverse component of the resistivity measured across the sample can be expressed as

$$
\rho_{x y}=\frac{h}{e^{2}} \frac{\sum_{i}\left(I_{i} / N_{i}\right)}{\sum_{i} I_{i}},
$$

where $I_{i}$ is the local current through the $i$-th QH puddle between the voltage probes, and $N_{i}$ its local filling factor. This yields a quantized value only provided all the $N_{i}$ 's are identical. This is no longer the case for $\nu>1$ : Eq. (14) generally describes a weighted average in which the weight of terms with $N_{i}>1$ is monotonically increasing with $\nu$. As a result $\rho_{x y} \sim h / \nu e^{2}$, as in the classical Hall regime. Along the critical line, this implies $\rho_{x y}^{c} \sim h / \nu_{c} e^{2}$. The transition points in the vicinity of integer values $N>$ 1 of $\nu_{c}$ correspond to the cases where a majority of the $\mathrm{QH}$ puddles consists of a unique filling factor $N$. They therefore characterize direct transitions from $N>1 \mathrm{QH}$ phases to the insulator.

The role of mixing with high LL does not only lead to violation of the universality of $\rho_{x x}^{c}$ : naturally, the behavior of $\rho_{x x}(\nu, T)$ across the transition is also modified. Indeed, in the high $B$ limit, isotherms $\rho_{x x}$ vs. $\nu$ can be fitted to a simple formula [3]

$$
\rho_{x x}=\frac{h}{e^{2}} \exp \left[\frac{-\Delta \nu}{\nu_{0}(T)}\right]
$$

where $\Delta \nu=\nu-\nu_{c}$ and $\nu_{0}(T) \approx \alpha T+\beta$. This formula exhibits a duality symmetry $\rho_{x x}(\Delta \nu)=\rho_{x x}^{-1}(-\Delta \nu)$, which is eventually violated in the lower $B$ regime. The analysis presented in this paper implies a specific modification of the above formula, which can be used as a test for the proposed transport scenario. As shown in Ref. [12], the simple exponential dependence on $\Delta \nu$ in Eq. (15) can be derived from the energy dependent expression Eq. (3) (with an effective activation temperature) assuming a parabolic shape of the barriers, such that $\left(\epsilon_{p}-\epsilon_{F}(\nu)\right) \propto(-\Delta \nu)$. Employing the same approximation in the moderately high $B$ regime, where the resistivity is given by Eq. (11), and relating the parameter $\Gamma_{p}$ to $\rho_{x x}^{c}$ through Eq. (12), the isotherms acquire the form

$$
\rho_{x x}(\nu) \approx \frac{\left(\rho_{x x}^{c}-h / e^{2}\right)}{2}+\frac{\left(\rho_{x x}^{c}+h / e^{2}\right)}{2} \exp \left[\frac{-\Delta \nu}{\nu_{0}(T)}\right] .
$$

In Fig. 2, a few such isotherms are plotted for a case where $\rho_{x x}^{c}$ is considerably elevated from $h / e^{2}$. Note that for $\Delta \nu>0, \log \rho_{x x}$ vs. $\Delta \nu$ curve upward compared to the straight line expected from the symmetric formula 




FIG. 2. The longitudinal resistivity (normalized in units of $\rho_{x x}^{c}$ ) on a $\log$ scale as a function of filling factor, computed from Eq. (16) for $\rho_{x x}^{c}=2.2 h / e^{2}$, and $\nu_{0}=0.079,0.11,0.125,0.15,0.175,0.2\left(\nu_{0}=0.079\right.$ is the steepest curve).

Eq. (15). A preliminary comparison with experimental data (e.g. Ref. [5]) confirms this behavior.

Finally, it is interesting to comment on the application of the transport model described here for transitions from the fractional QH state $1 / 3$ to the insulator. Experimentally [2], the transport data exhibit the same behavior as near the integer transitions: e.g., Eq. (15) is obeyed replacing $\nu$ by an effective filling factor of composite Fermions. This suggests that a similar model describes here the d.c. transport properties of composite Fermions. In particular, high LL of Fermions correspond to the hirarchy fractions $2 / 5,3 / 7$ etc. In analogy with the integer case, occupation of localized high LL Fermion states would imply the formation of incompressible regions in the disordered sample at these filling fractions. However, in contrast with the integer case, the energy gap supporting such regions is tiny. As a result, a mechanism for enhancing the resistance by backscattering through localized high LL states is expected to be practically absent. This possibly explains the observation that the duality symmetry in $\rho_{x x}$, as well as the universality of $\rho_{x x}^{c}$ that comes with it, are better manifested by data near the fractional transitions.

I thank A. Auerbach, M. Hilke, A. Kamenev, D. Shahar, and S. Sondhi for useful conversations. This work was supported by grant no. 96-00294 from the United States-Israel Binational Science Foundation (BSF), Jerusalem, Israel.
[1] For a review and extensive references, see A. M. M. Pruisken in The Quantum Hall Effect, Eds. R. E. Prange and S. M. Girvin (Springer-Verlag, New York, 1986); S. Das Sarma in Perspectives in the Quantum Hall Effect, Eds. S. Das Sarma and A. Pinczuk (John Wiley and Sons, 1997); S. L. Sondhi, S. M. Girvin, J. P. Carini and D. Shahar, Rev. Mod. Phys. 69, 315 (1997).

[2] D. Shahar, D. C. Tsui, M. Shayegan, R. N. Bhatt and J. E. Cunningham, Phys. Rev. Lett. 74, 4511 (1995); D. Shahar, D. C. Tsui, M. Shayegan, E. Shimshoni and S. L. Sondhi, Science 274, 589 (1996).

[3] D. Shahar, M. Hilke, C. C. Li, D. C. Tsui, S. L. Sondhi and M. Razeghi, Solid State Comm. 107, 19 (1998).

[4] D. Shahar, D. C. Tsui, M. Shayegan, J. E. Cunningham, E. Shimshoni an d S. L. Sondhi, Solid State Comm. 102, 817 (1997); M. Hilke, D. Shahar, S. H. Song, D. C. Tsui, Y. H. Xie and Don Monroe, Nature 395, 673 (1998); M. V. Yakunin, Yu. G. Arapov, O. A. Kuznetsov and V. N. Neverov, JETP Lett. 70, 301 (1999).

[5] M. Hilke, D. Shahar, S. H. Song, D. C. Tsui, Y. H. Xie and Don Monroe, Phys. Rev. B 56, R15525 (1997).

[6] Y. Hanein, D. Shahar, H. Shtrikman, J. Yoon, C. C. Li and D. C. Tsui, Nature 400, 735 (1999).

[7] M. Hilke, D. Shahar, S. H. Song, D. C. Tsui and Y. H. Xie, to be published in Phys. Rev. B (preprint condmat/9906212).

[8] D. E. Khmel'nitzkii, JETP Lett. 38, 552 (1983); S. Kivelson, D. H. Lee and S. C. Zhang, Phys. Rev. B 46, 2223 (1992).

[9] D. N. Sheng and Z. Y. Weng, cond-mat/9906261.

[10] M. Hilke, private communication.

[11] E. Shimshoni and A. Auerbach, Phys. Rev. B 55, 9817 (1997).

[12] E. Shimshoni, A. Auerbach and A. Kapitulnik, Phys. Rev. Lett. 80, 3352 (1998).

[13] Y. Meir, Phys. Rev. Lett. 83, 3506 (1999); Y. Meir, condmat/9912423.

[14] M. Büttiker, Phys. Rev. Lett. 57, 1761 (1986); M. Büttiker, Phys. Rev. B 38, 9375 (1988).

[15] V. Ambegaokar, B. I. Halperin and J. S. Langer, Phys. Rev. B 4, 2612 (1971).

[16] The weak links to higher LL channels are located at arbirary distances from the nearest junctions in the network, which are typically of the same order as the size of a puddle.

[17] The actual crossover value of $\nu_{c}$ is slightly higher than 1 for $\nu_{h}>2$; see, e.g., Ref. []. 